\title{
Nutraceuticals and peripheral glial cells: a possible link?
}

\section{Keywords}

Satellite glial cells; Nutraceuticals; Resveratrol; Curcumin; Quercetin; Diabetes mellitus; Nerve injury; Peripheral nervous system

\section{Introduction}

The term "nutraceutical" was coined in 1989 by the Foundation for Innovation in Medicine (New York, NY, USA) as "any food substance or part of a food that provides specific medical or health benefits beyond their basic nutritional value" [1], including prevention and treatment of a wide variety of diseases such as cancer [2], cardiovascular diseases [3-5], obesity [6], diabetes [7, 8], Alzheimer's and Parkin- son's diseases [9-12] or eye disorders [13], among many others. Considering their preventive and therapeutic potential and the current globalization context, constantly in search for sustainable solutions for economy, environment and health [14-16], the number of studies reporting the modulating effects of nutraceuticals on the different functions of the body and, particularly, on the elements of the nervous system is rapidly increasing. Studies are mainly focused on neurons and glial cells $[9,11,17-21]$.

Next to the neurons, glial cells constitute a second branch of the nervous system. They are present in both the central (CNS: astroglia, oligodendroglia and microglia) and peripheral nervous system (PNS: Schwann cells, olfactory ensheathing cells, satellite glial cells, enteric glial cells) [22]. At first, glia was considered structural support for neurons in the nervous system. Still, in recent years their functions have been more deeply investigated, and they turned out to be as important as nerve cells, because of their role in all aspects of neural functions [23]. Other glial cells, like microglia, Schwann cells or enteric glial cells have been intensely studied, even in aspects like the modulation of their functions by nutraceuticals [9, 11, 20, 24-35], but satellite glial cells (SCGs) have received less attention. However, SGCs are increasingly recognized for their essential roles in physiology and disease $[36,37]$.

\section{Satellite glial cells}

Satellite glial cells are present in sensory ganglia (dorsal root ganglia, DRG, trigeminal ganglia, TG) and sympathetic and parasympathetic ganglia. Their main features are summarized in Table 1. We will focus on the sensory neurons and surrounding glial cells present in the DRG and TG, because, on the one hand, data concerning SGCs physiology in the autonomic nervous system is limited, and, on the other hand, data regarding the modulatory effects of nutraceuticals on SGCs are almost only available for DRG and TG. 
Table 1. Characteristics of satellite glial cells.

\begin{tabular}{ll}
\hline Feature & Satellite glial cells \\
\hline $\begin{array}{l}\text { Morphology } \\
\text { Subtypes }\end{array}$ & Laminar, normally devoid of processes \\
Location & Not recognized \\
& Dorsal root ganglia, trigeminal ganglia, other peripheral ganglia \\
& GFAP \\
& Kir4.1 \\
Proposed markers & Cadherin 19 \\
& GS \\
& SK3 \\
Adjacent cells coupling & Gap junction coupling \\
& Able to release pro-inflammatory cytokines (i.e., IL-1 $\beta$, TNF- $\alpha$ ) \\
Activation & Activation of MAPK pathways, i.e., phosphorylation of p38 and ERK \\
& Enhanced expression of glial cells markers \\
Involved in & Chronic pain (inflammatory, neuropathic, visceral) \\
\hline Abbreviations: ERK, extracellular signal-regulated kinase; GFAP, glial fibrillary acidic protein; GS, \\
glutamine synthetase; IL, interleukin; Kir4.1, inwardly rectifying potassium channel, type 4.1; MAPK, \\
mitogen-activated protein kinase; SK3, small conductance calcium-activated potassium channel; TNF- \\
$\begin{array}{l}\alpha, \text { tumor necrosis factor } \alpha .\end{array}$
\end{tabular}

Satellite glial cells share similarities with Schwann cells, as they are derived from the neural crest and occur in the PNS. Nevertheless, SGCs also exhibit common features with astrocytes, as like these cells, they express several proteins recognized as glial cell markers: glial fibrillary acidic protein (GFAP), and glutamine synthetase (GS). Furthermore, SGCs are coupled by gap junctions similarly to glia in the CNS and the enteric nervous system (ENS) [36, 38]. However, unlike astrocytes, SGCs have laminar-shaped cell bodies and lack processes. A unique function of SGCs is that they wrap around and compose envelopes around the neural cell bodies. Neural cells and surrounding SGCs constitute a distinct functional unit (neuron-glial unit). Usually, a single neuron is enveloped by a few SGCs, but there may be exceptions: occasionally in the DRG, two neurons share a common SGCs envelope, creating a 'cluster'. The extracellular space between the neuron and SGCs measures only $20 \mathrm{~nm}$ enabling rapid bidirectional communication [38].

Studies on the role of SCGs in physiology and pathophysiology are limited due to the lack of specific molecular tools. They were recognized mainly according to their morphology and location. Several markers of SGCs were distinguished: i.e., GFAP [39], inwardly rectifying potassium channel (Kir4.1) [40], cadherin 19 [41], GS [42], calcium activated potassium channel (SK3; small conductance calciumactivated potassium channel 3) [43, 44]. It remains extremely difficult to purify SGCs and investigate their biology at the molecular level, because all above markers do not appear specific to SGCs. For example, GS at the immunostaining level seems specific. Still, it does not label all SGCs, while at messenger ribonucleic acid (RNA) expression level, in the DRG, GS is expressed by many cell types, not only SGCs [45].

\section{Satellite glial cells activation}

There is emerging evidence that the morphology and function of SGCs change under the influence of harmful fac- tors, such as nerve injury or inflammation [46-49]. The response of SGCs to peripheral nerve injury is rapid and takes place within 4 hours. This reaction peaks at 1 week and begins to alleviate after 3 weeks [48-50].

Activated SGCs synthesize and release pro-inflammatory cytokines (i.e., interleukin (IL)- $1 \beta$, tumor necrosis factor (TNF)- $\alpha$ ) and contribute to pain reaction through the activation of several signaling pathways, i.e., mitogen-activated protein kinase (MAPK) pathways [51].

The family of MAPK includes extracellular signalregulated kinase 1 and 2 (ERK1 and ERK2), p38, and c-Jun Nterminal kinases (JNK). MAPK signaling pathway maintains an important role in intracellular signaling in neurons during persistent pain. In several studies, it was evidenced that nerve fiber injury results in augmented phosphorylation (activation) of p38 in SGCs of DRG $[52,53]$, while the peripheral inflammation of temporomandibular joint results in increased activation of this pathway in SGCs of TG [54]. Noteworthy, axonal injury leads to the phosphorylation of ERK in SGCs in DRG [55], whereas the inflammation of the temporomandibular joint promotes activation of ERK in SGCs of TG [54]. MAPK pathways are inactivated by phosphatases (MAPK phosphatase 1, 2, 3, MKP). It was assessed that the expression of MKP1, MKP2 and MKP3 rapidly increases in SCGs of TG during inflammation [54].

Following nerve injury, SGCs proliferation increases, and these cells display increased expression of GFAP [56] and enhanced production of cytokines (IL- $1 \beta$, IL- 6, TNF- $\alpha$ ) $[57,58]$. Furthermore, SGCs can release glial mediators (i.e., basic fibroblast growth factor 4, (bFGF4) or matrix metalloproteinase-2, (MMP-2)) which may activate other glial cells via paracrine/autocrine mechanisms [59].

Finally, besides previously mentioned changes, adenosine triphosphate (ATP) sensitivity increases and purinergic signaling is altered. 


\section{Effects of nutraceuticals on satellite glial cells}

Thus far, the possible modulatory role of nutraceuticals on SGCs has only been evaluated for a few compounds, whose key properties are shown in Table 2. Furthermore, their effects have only been analyzed in animal models and cell cultures in a few reports (see Table 3 (Ref. [60-70]) and the text below for references).

In 1999 it was observed that vitamin E deficiency in rats caused the proliferation of SGCs, shown by immunohistochemical methods [60]. Vitamin E deficiency causes neurodegenerative processes in the DRG [61] that would induce a response in SGCs. Taking this into account, it was postulated that vitamin $\mathrm{E}$ could function as an exogenous control for this proliferation in pathological conditions. Further research using disease models is needed to clarify if administering vitamin $\mathrm{E}$ would have a beneficial role and whether, in addition to proliferation, other mechanisms (purinergic signaling, nitric oxide or cytokine release...) are involved in the suggested beneficial effect of vitamin $\mathrm{E}$.

The other nutraceuticals whose effects on SGCs from DRG and TG have been described seem to do so through antioxidant mechanisms or interference with purinergic signaling. These will be summarized in the following sections.

\subsection{Nutraceuticals acting through antioxidant mechanisms}

Nutraceuticals with antioxidant capacities have been evaluated in several studies. Indeed, some diseases exert damaging effects on SGCs due to free radicals and nutraceuticals seem to exert beneficial effects in these conditions.

One such antioxidant compound is $\mathrm{N}$-acetylcysteine (NAC), a safe and commonly used drug in the clinics, also present in some fruits and vegetables [71]. The main antioxidant effect is due to the ability of NAC to act as a reduced glutathione (GSH) precursor; GSH is a well-known direct antioxidant and a substrate of several antioxidant enzymes. Moreover, in some conditions where a significant depletion of endogenous cysteine (Cys) and GSH occurs, NAC can act as a direct antioxidant for some oxidant species such as $\mathrm{NO}_{2}$. Thus, NAC would act as a Cys donor. The antioxidant activity of NAC could also be due to its effect in breaking thiolated proteins, thus releasing free thiols and reduced proteins. Besides being involved in these antioxidant mechanisms, the disulphide breaking activity of NAC also explains its wellknown mucolytic activity which is due to its effect in reducing heavily cross-linked mucus glycoproteins [72]. All these properties justify its already extended clinical use to counteract acetaminophen-induced hepatotoxicity and to clear airways [73] and the search for new potential uses, like treatment of peripheral pain, which involves SGCs.

An investigation on postoperative hyperalgesia induced by remifentanil in Sprague-Dawley rats focused on the usefulness of NAC in modulating the action of MMP-9 metalloproteases, which were up-regulated and activated by remifentanil in the DRGs [62]. Matrix metalloproteases are a group of calcium-dependent enzymes responsible for the cleavage of extracellular matrix proteins and cytokines [74]. The excessive proteolytic activity of MMP-9 damages DRGs and may increase neuronal transmission and produce neuropathic pain by stimulating the activation of microglia [75]. MMP-9 is mainly expressed in neurons that co-express mu opioid receptors (MOR) [76] and causes maturation of proinflammatory cytokines through the cleavage of IL- $1 \beta$. Acting as a Cys donor, NAC prevented the cysteine residue present in MMP-9 from being oxidized, which is vital for its activation. Thus, it was demonstrated that NAC inhibits the cleavage of IL-1 $\beta$ in DRGs and suppresses glial activation and neuronal excitability induced by remifentanil, alleviating hyperalgesia [62].

In Sprague-Dawley rats, Hart et al. [63] investigated the effects of systemic NAC upon cell death within DRG, which characterizes many peripheral neuropathies, particularly those of traumatic etiology. NAC was applied after peripheral nerve injury, and its neuroprotective potential was analyzed. Specifically, the effect of treatment upon cell survival was quantified using terminal deoxynucleotidyl transferase-mediated dUTP nick end labeling (TUNEL), a method for detecting apoptotic deoxyribonucleic acid (DNA) fragmentation, widely used to identify and quantify apoptotic cells [77]. TUNEL positive cells were more numerous in axotomized ganglia, and NAC application provoked a reduction in the number of TUNEL positive glial cells. Additionally, mitochondrial architecture, usually damaged in this kind of neuropathies, was preserved. In vivo treatment with NAC might therefore increase glial glutathione production, potentially protecting glia not only directly but indirectly, through neuroprotection secondary to increased neuronal glutathione levels.

Curcumin, a spice obtained from the rhizome of turmeric, is another compound with antioxidant action, well-known to act as a free radical scavenger $[78,79]$. Pathological processes such as sciatic nerve crush lead to poor function of the affected limb and induce histological changes including neuronal loss, as already mentioned. Norafshan et al. [64] studied this process in Sprague-Dawley rats and found a reduction in the number of SGCs in the lumbar 5 (L5) DRG. The damaging insult produces a period of ischemia with the release of chemical mediators, an increase in vascular permeability and a deficiency in the blood-nerve barrier [80], with edema, inflammation and metabolic disability in the cells [81, 82]. All these events favor the production of toxic oxygen metabolites such as superoxide anions, hydrogen peroxide and hydroxyl radicals due to the infiltration of polymorphonuclear leukocytes into the lesioned areas. These free radicals and cytokines released by neutrophils are responsible for cell damage. SGCs damage (measured histologically in the DRG and the associated signs of painful neuropathy (measured in vivo) were less intense with curcumin treatment, probably due to the reduction of toxic oxygen-derived metabolites, although this mechanism was not directly evaluated. 
Table 2. General characteristics of nutraceuticals studied on satellite glial cells.

\begin{tabular}{|c|c|c|c|c|}
\hline Nutraceutical & Characteristics & Structure & Source & Properties \\
\hline \multirow{2}{*}{ Vitamin E } & \multirow{2}{*}{ Fat-soluble vitamin } & & Plant-derived oils, nuts, seeds, fruits, and vegetables & Antioxidant \\
\hline & & & Available as a dietary supplement & Modulation of proliferation \\
\hline $\mathrm{N}$-acetylcysteine & Amino acid derivative & & Supplement form of cysteine & Antioxidant, mucolytic \\
\hline \multirow[t]{2}{*}{ Curcumin } & \multicolumn{2}{|l|}{ Flavonoid polyphenol } & Produced by plants of the Curcuma longa species & Anti-inflammatory, antioxidant, anticancer, antiapoptotic \\
\hline & \multirow[t]{2}{*}{ Flavonoid polyphenol } & & Fruits and vegetables & Anti-oxidant, anti-inflammatory, and anti-nociceptive \\
\hline & & & Available as a dietary supplement & \\
\hline \multirow[t]{2}{*}{ Osthole } & \multirow[t]{2}{*}{ O-methylated coumarin } & & Found in a variety of plants & Anti-inflammatory and antioxidant \\
\hline & & & Available as a dietary supplement & \\
\hline \multirow[t]{2}{*}{ Resveratrol } & \multirow[t]{2}{*}{ Non-flavonoid polyphenol } & & Concentrated mostly in the skins and seeds of grapes and berries & Anti-inflammatory and anti-nociceptive \\
\hline & & & Available as a dietary supplement & \\
\hline
\end{tabular}

Molecules were made using http://biomodel.uah.es/en/DIY/JSME/draw.es.htm. 
Table 3. Effects of nutraceuticals on satellite glial cells.

\begin{tabular}{|c|c|c|c|c|}
\hline Nutraceutical & Species & Pathology & Key findings & References \\
\hline \multirow{3}{*}{ Vitamin E } & \multirow{2}{*}{ Sprague-Dawley rats } & \multirow{2}{*}{ Vitamin E deficiency } & Vitamin E-deficient rats present a faster turnover in SGCs & \multirow{2}{*}[60]{} \\
\hline & & & Control of SGCs proliferation & \\
\hline & Rhesus monkeys & Vitamin E deficiency & Neuropathologic lesions in DRG & [61] \\
\hline \multirow{5}{*}{$\mathrm{N}$-acetylcysteine } & \multirow{3}{*}{ Sprague-Dawley rats } & \multirow{3}{*}{ Postoperative hyperalgesia } & Attenuates mechanical allodynia and thermal hyperalgesia & \multirow{3}{*}{ [62] } \\
\hline & & & Suppresses MMP-9 activation & \\
\hline & & & Inhibits the cleavage of IL- $1 \beta$ in DRGs & \\
\hline & \multirow{2}{*}{ Sprague-Dawley rats } & \multirow{2}{*}{ Nerve injury } & Neuroprotection & \multirow{2}{*}{ [63] } \\
\hline & & & Mitochondrial preservation in SGCs & \\
\hline \multirow{3}{*}{ Curcumin } & Sprague-Dawley rats & Sciatic nerve crush & Neuroprotective effects on the DRG, prevents SGC loss & [64] \\
\hline & \multirow{2}{*}{ STZ treated Sprague-Dawley rats } & \multirow{2}{*}{ Diabetes mellitus } & Prevents mechanical and thermal hyperalgesia & \multirow{2}{*}{ [65] } \\
\hline & & & Prevents upregulation of P2Y12 receptor on SGC & \\
\hline \multirow{4}{*}{ Quercetin } & \multirow{2}{*}{ Sprague-Dawley rats and C6 rat glioma cell line } & \multirow{2}{*}{ Spared nerve injury } & Attenuates neuropathic pain & \multirow{2}{*}{ [66] } \\
\hline & & & GFAP inhibition in the L5 DRGs & \\
\hline & \multirow{2}{*}{ STZ-treated Sprague-Dawley rats } & \multirow{2}{*}{ Diabetes mellitus } & Attenuates neuropathic pain & \multirow{2}{*}{ [67] } \\
\hline & & & Inhibits P2X4 receptor-mediated p38MAPK activation & \\
\hline \multirow{3}{*}{ Osthole } & \multirow{3}{*}{$\begin{array}{l}\text { STZ treated Sprague-Dawley rats and HEK293 } \\
\text { cells transfected with P2X4 plasmid }\end{array}$} & \multirow{3}{*}{ Diabetes Mellitus } & Alleviates mechanical and thermal hyperalgesia through P2X4 receptor & \multirow{3}{*}{ [68] } \\
\hline & & & Reduces up-regulation of IL-1 $\beta$, TNF- $\alpha$, BDNF and phosphorylated-p38MAPK & \\
\hline & & & Enhance the down-regulation of IL-10 & \\
\hline \multirow[t]{2}{*}{ Resveratrol } & $\begin{array}{l}\text { Gp } 120 \text { treated Sprague-Dawley rats and HEK293 } \\
\text { cells transfected with P2X7 plasmid }\end{array}$ & HIV-associated neuropathic pain & Relieves mechanical hyperalgesia inhibiting the P2X7 receptor on SGCs & [69] \\
\hline & Sprague-Dawley rats & Chronic constriction injury & Suppresses the transmission of neuropathic pain mediated by the P2X7 receptor in SGCs of DRG & [70] \\
\hline
\end{tabular}




\subsection{Nutraceuticals interfering with purinergic signaling}

Curcumin may also act through purinergic receptors. Specifically, this mechanism of action has been shown for curcumin in the field of diabetes mellitus and the neuropathic pain that it entails. Jia et al. [65] used the classical type 1 diabetes mellitus model, induced by streptozotocin (STZ) injection, in adult Sprague-Dawley rats, where they tested the effect of curcumin-loaded nanoparticles, since the low in vivo metabolic stability of this compound and its low bioavailability make it necessary to find more efficient forms of administration [83]. The G protein-coupled type 2 (P2) purinergic receptor P2Y12 is expressed in SGCs and plays an important role in transmitting pain signals. These P2Y12 receptors are increased in SGCs of animals with diabetes that suffer thermal hyperalgesia, associated with increased production of the pro-inflammatory cytokine IL- $1 \beta$ and an increased expression of Cx43 (connexin 43) in DRG [65]. As also previously mentioned, gap junctions between SGCs play an important role in neuronal excitability and contribute to the induction and maintenance of pain. Thus, the upregulation of P2Y12 in SGCs and of IL- $1 \beta$ and Cx43 in the DRG indicates activation of SGCs. Treatment with nanoparticulate curcumin, probably through the prevention of $\mathrm{P} 2 \mathrm{Y} 12$ receptor up-regulation, inhibited the activation of SGCs with a consequent decrease in thermal and mechanical hyperalgesia [65].

Inhibition of other types of purinergic receptors 2 (P2X4 and P2X7) appears to be the mechanism of action by which other nutraceuticals such as quercetin, resveratrol, and osthole exert their effects on SGCs.

Quercetin is a flavonoid widely distributed in fruits and vegetables such as onions, blueberries, or apples with antioxidant, anti-inflammatory and antinociceptive properties [84]. It is also a supplement for the alternative treatment of allergies, asthma, arthritis, hypertension, or neurodegenerative disorders [85-88]. Using a rat model that developed neuropathic pain as a consequence of spared nerve injury, it was found that the administration of quercetin before surgery attenuated mechanical allodynia and inhibited GFAP expression (measured by Western blot) and labeling (measured immunohistochemically) in L5 DRG [66]. After the peripheral nerve damage, SGCs changed their number and function, revealing an increase in gap junctions that enhanced intercellular coupling of the SGCs and decreased their membrane resistance, causing pain. In addition, SGCs upregulated the production of pro-inflammatory cytokines such as TNF- $\alpha$. The application of quercetin in this model caused the inhibition of SGCs, as indicated by the suppression of GFAP labeling, thus contributing to pain reduction [66]. Interestingly, in the same report, the authors demonstrated a similar, dosedependent effect of quercetin on GFAP levels, elevated by IL-6 in a C6 rat glioma cultured cell line used as a cellular astrocyte model [66]. These results suggest that quercetin may have a broad glioprotective action.

Using STZ-induced diabetic Sprague-Dawley rats, the mechanism of action of quercetin was studied in depth [67].
Thus, combining real-time reverse-transcription polymerase chain reaction (RT-PCR) for P2X 4 receptor, Western blot for P2X4 and p38 MAPK, immunofluorescence for P2X 4 and GFAP and molecular docking techniques (computer simulation tools used to predict the binding mode of a ligand in the active site of a protein), quercetin appeared to act through the inhibition of P2X4 receptors located on SGCs. Upregulation of these nociceptive ion channels contributes to developing and maintaining pain-related symptoms of diabetes mellitus induced by STZ. These studies showed the ability of quercetin to bind the P2X4 receptor, effectively decreasing its upregulation in DRG SGCs, and consequently inhibiting the activation of p38 MAPK mediated by this receptor, which led to thermal and mechanical hyperalgesia alleviation in diabetic rats.

The $\mathrm{P} 2 \mathrm{X} 4$ receptor also appears to be behind the beneficial effects of osthole, a component extracted from the seeds of the Cnidium monnieri $(L)$ cusson plant with anti-inflammatory and antioxidant properties in the diabetic neuropathy that was induced in male Sprague-Dawley rats after STZ injection [68]. Again, different techniques were used, including RT-PCR for P2X4 detection, Western blot analysis of GFAP, P2X4, p38 MAPK, p-P38 MAPK and brain-derived neurotrophic factor (BDNF), and immunofluorescence labeling of P2X4 receptor and GFAP. Osthole decreased the upregulation of this receptor and the activation of SGCs in the DRGs that occurs during diabetes mellitus, followed by downregulation of BDNF, IL- $1 \beta$ and p38 MAPK. In this way, thermal and mechanical hyperalgesia would be inhibited. To confirm the involvement of $\mathrm{P} 2 \mathrm{X} 4$ receptors in the effect of quercetin, the authors performed whole-cell patch-clamp recordings of HEK293 cells transfected with the pEGFP-hP2X4 plasmid. ATP activated HEK293 cells expressing P2X4 receptors, and quercetin significantly inhibited ATP-activated current in these cells, strongly suggesting that quercetin may relieve pain behaviors in diabetic rats by inhibiting P2X4 signaling [68].

As already mentioned, resveratrol is a naturally occurring polyphenolic compound found in peanuts, blackberries, grapes, and red wine with antinociceptive and antiinflammatory effects [89]. Two studies using SpragueDawley rats have analyzed its mechanism of action in two different animal models: a chronic pain model caused by administration of gp120, derived from the human immunodeficiency virus (HIV) [69]; and a neuropathic pain model caused by chronic constriction injury [70]. In both cases, resveratrol seemed to act through the inhibition of $\mathrm{P} 2 \mathrm{X} 7$ purinergic receptors located on SGCs of DRG, which are related to the induction and maintenance of neuropathic and inflammatory pain. In both animal models, treatment with resveratrol reduced pain transmission, suppressing the expression of the P2X7 receptor and preventing an elevation of the pain threshold. The mechanism by which it relieves pain includes the inhibition of P2X7 and GFAP in SCGs and the consequent reduction in the release of cytokines such as TNF- $\alpha$ 


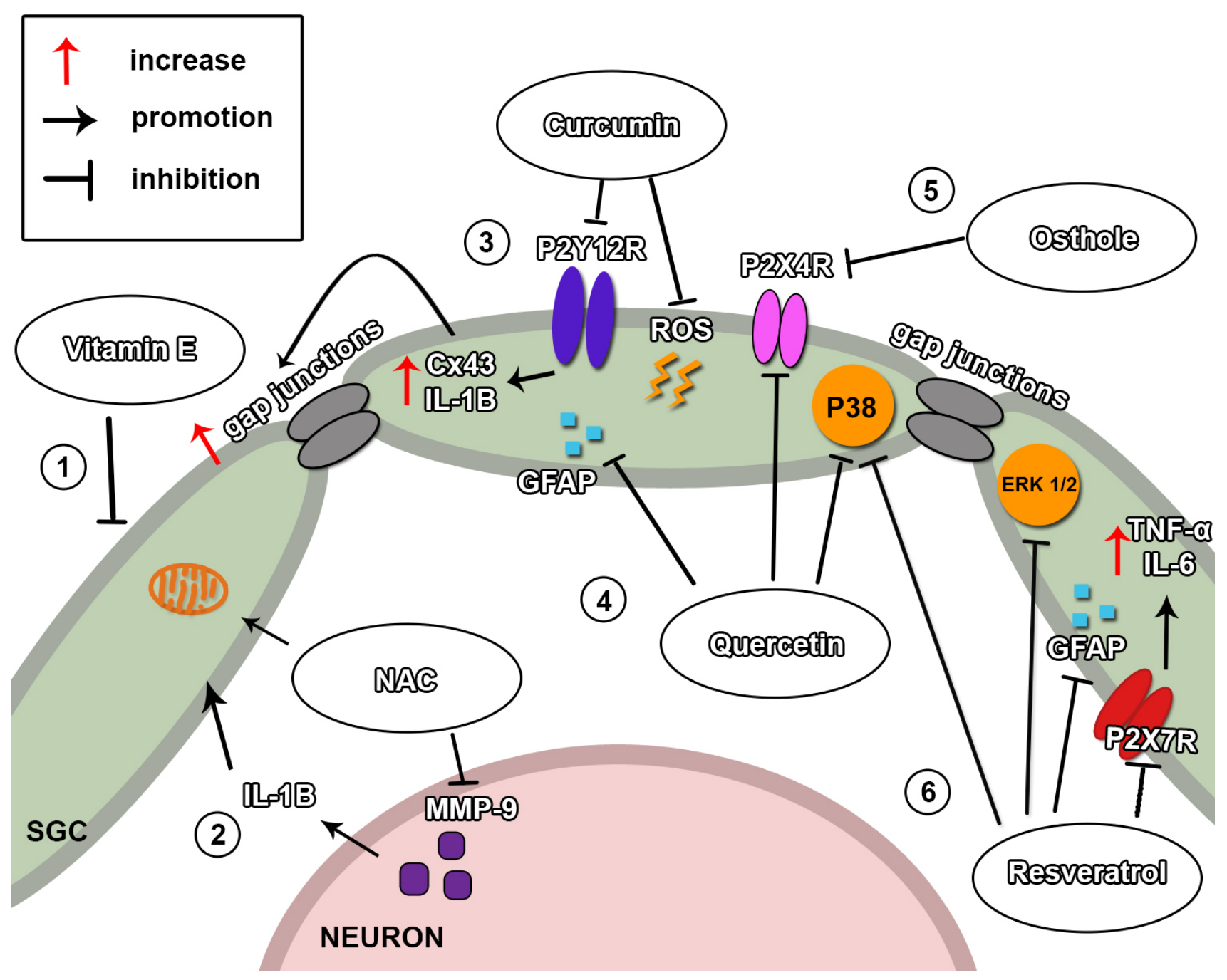

Fig. 1. Nutraceuticals and their mechanisms of action on the satellite glial cells. Vitamin E controls the proliferation of SGCs, but further research is needed to elucidate the exact mechanism of its action (1). NAC modulates the action of MMP-9, expressed on neurons in DRG, inhibits IL-1B cleavage and enhances the mitochondrial preservation in SGCs (2). The P2Y12 receptor, IL-1B and Cx43 (gap junctions building component), following nerve injury, are up-regulated. The proposed mechanism of action of curcumin involves the prevention from P2Y12 receptor up-regulation, resulting in the decrease of IL-1B production and excessive SGCs activation associated with the increment of the number of gap junctions connecting adjacent SGCs. Curcumin also exerts antioxidant effects by ROS scavenging (3). Quercetin decreases the up-regulation of GFAP and P2X4 receptors on the surface of SGCs (which results from SGCs activation), and consequently, blocks the activation of p38 MAPK (4). Osthole downregulates the P2X4 receptors and thus inhibits the activation of SGCs (5). Finally, resveratrol relieves mechanical hyperalgesia and suppresses the transmission of neuropathic pain through the inhibition of $\mathrm{P} 2 \mathrm{X} 7 \mathrm{receptor}$ and ERK1/2 and inhibits GFAP up-regulation (6). Abbreviations: Cx43-connexin 43; ERK-extracellular signal-regulated kinase; GFAP-glial fibrillary acidic protein; IL-1B-interleukin 1B; MMP-9-matrix metallopeptidase; NAC-N-acetylcysteine; P2X7R, P2X4R, P2Y12R-purinergic receptors 2; P38 MAPKp38 mitogen-activated protein kinases; ROS-reactive oxygen species; SGC-satellite glial cell; TNF- $\alpha$-tumor necrosis factor $\alpha$.

and IL-6 that would block the receptor's downstream signals ERK1/2 and p38 MARK, leading to pain attenuation. ATP is a signaling molecule that can activate $\mathrm{P} 2 \mathrm{X} 7$ receptors on SCGs, mediating pain, and other cells, like macrophages, mediating inflammation [90]. As in the previous studies with osthole, HEK293 cells were transfected with the P2X7 plasmid and the ATP-activated currents in these cells were recorded by whole-cell patch clamping. It was shown that resveratrol significantly inhibited those ATP-activated currents. These data further suggest that resveratrol relieves pain in these models by acting on the P2X7.

\section{Conclusions}

Here, we have reviewed the physiopathological roles of satellite glial cells, a relatively understudied type of peripheral glial cells, as well as the research evaluating the effects of different nutraceuticals and food components on them (Tables 2,3), and the mechanisms involved (Fig. 1).

In general, reports on nutraceuticals' modulatory effects on these glial cells are relatively scarce. They might be somehow biased since only a few research groups have been actively involved in these studies (i.e., the same Chinese group from Nanchang University performed 5 out of 11 studies), 
in part due to the technical difficulties in reliably identifying these cells. The findings, mainly obtained in rodent models, seem to indicate that compounds like NAC, curcumin, quercetin, osthole, and resveratrol may exert beneficial effects in the treatment of neuropathic pain associated with different pathologies as varied as traumatic injury, diabetes, HIV infection, in which alterations of satellite glial cells occur. Furthermore, antioxidant mechanisms and interference with purinergic signaling were identified as underlying the effects on these cells of the nutraceuticals studied.

Although the results obtained thus far are encouraging, the research in this field is clearly in its infancy. The modulatory role of other nutraceuticals on satellite glial cell activity and their mechanisms of action need to be investigated. Among these, one important but neglected issue is the possible role of intestinal microbiota, which, on the one hand, release microbial-associated molecular patterns with potential systemic effects, and, on the other, actively metabolize nutritional components and drugs in the intestines, adding complexity to the effects food-derived nutraceuticals and their metabolites can cause both in the gut and distantly, after absorption. This has been previously addressed for other glial cell types (for example, [91, 92] illustrate the relevance of the glia-microbiota-nervous system axis) and should also be done in this case. Furthermore, the effects of nutraceuticals and their mechanisms of action need to be evaluated in additional preclinical models (i.e., chemotherapy-induced neuropathic pain, visceral pain, nerve regeneration...), and the results from these studies should be validated in robust clinical trials.

Hopefully, further research will soon define the connections between nutraceuticals and satellite glial cells as a possible target to treat, prevent or reduce their alterations associated with the different disorders in which they are involved, particularly chronic pain.

\section{Abbreviations}

ATP, adenosine triphosphate; BDNF, brain derived neurotrophic factor; bFGF, basic fibroblast growth factor; CNS, central nervous system; Cx43, connexin 43; Cys, cysteine; DNA deoxyribonucleic acid; DRG, dorsal root ganglion, dorsal root ganglia; ENS, enteric nervous system; ERK, extracellular signal-regulated kinase; GFAP, glial fibrillary acidic protein; GS, glutamine synthetase; GSH, reduced glutathione; HIV, human immunodeficiency virus; IL, interleukin; JNK, c-Jun N-terminal kinases; Kir, inwardly rectifying potassium channel; L5, lumbar 5; MAPK, mitogen-activated protein kinase; MKPs, MAPK phosphatases; MMP, matrix metalloproteinase; MOR, mu opioid receptors; NAC, N-acetylcysteine; $\mathrm{P} 2$, purinergic receptor 2; PNS, peripheral nervous system; RNA, ribonucleic acid; SGC, satellite glial cell; RT-PCR, real-time reverse-transcription polymerase chain reaction; SK3, small conductance calcium-activated potassium channel 3; STZ, streptozotocin; TG, trigeminal ganglia; TNF, tumor necrosis factor; TUNEL, terminal deoxynucleotidyl transferase-mediated dUTP nick end labeling.

\section{Author contributions}

Conceptualization: RA. Writing-original draft preparation: LLG, AS, MZ. Writing-review and editing RA. All authors have read and agreed to the published version of the manuscript.

\section{Ethics approval and consent to participate}

Not applicable.

\section{Acknowledgment}

Not applicable.

\section{Funding}

This research was funded by Ministerio de Ciencia, Innovación y Universidades (grant number PID2019-111510RBI00 to RA) and by National Science Center-SONATA 15 (number UMO-2019/35/D/NZ7/02830 to MZ).

\section{Conflict of interest}

The authors declare no conflict of interest.

\section{References}

[1] Helal NA, Eassa HA, Amer AM, Eltokhy MA, Edafiogho I, Nounou MI. Nutraceuticals' Novel Formulations: the Good, the Bad, the Unknown and Patents Involved. Recent Patents on Drug Delivery \& Formulation. 2019; 13: 105-156.

[2] Prasad S, Gupta SC, Tyagi AK. Reactive oxygen species (ROS) and cancer: Role of antioxidative nutraceuticals. Cancer Letters. 2017; 387: 95-105.

[3] Ghaffari S, Roshanravan N. The role of nutraceuticals in prevention and treatment of hypertension: an updated review of the literature. Food Research International. 2020; 128: 108749.

[4] Borghi C, Cicero AF. Nutraceuticals with a clinically detectable blood pressure-lowering effect: a review of available randomized clinical trials and their meta-analyses. British Journal of Clinical Pharmacology. 2017; 83: 163-171.

[5] Malekmohammad K, Sewell R, Rafieian-Kopaei M. Antioxidants and Atherosclerosis: Mechanistic Aspects. Biomolecules. 2019; 9: 301.

[6] Cuevas-Sierra A, Ramos-Lopez O, Riezu-Boj JI, Milagro FI, Martinez JA. Diet, Gut Microbiota, and Obesity: Links with Host Genetics and Epigenetics and Potential Applications. Advances in $\mathrm{Nu}$ trition. 2019; 10: S17-S30.

[7] Valdés-Ramos R, Guadarrama-López AL, Martínez-Carrillo BE, Benítez-Arciniega AD. Vitamins and Type 2 Diabetes Mellitus. Endocrine, Metabolic \& Immune Disorders Drug Targets. 2015; 15: 54-63.

[8] Salgaço MK, Oliveira L, Costa GN, Bianchi F, Sivieri K. Relationship between gut microbiota, probiotics, and type 2 diabetes mellitus. Applied Microbiology and Biotechnology. 2019; 103: 9229_ 9238.

[9] Sawikr Y, Yarla NS, Peluso I, Kamal MA, Aliev G, Bishayee A. Neuroinflammation in Alzheimer's Disease: The Preventive and Therapeutic Potential of Polyphenolic Nutraceuticals. Advances in Protein Chemistry and Structural Biology. 2017; 108: 33-57.

[10] Szczechowiak K, Diniz BS, Leszek J. Diet and Alzheimer's dementia - Nutritional approach to modulate inflammation. Pharmacology Biochemistry and Behavior. 2019; 184: 172743.

[11] McCarty MF, Lerner A. Nutraceuticals Targeting Generation and Oxidant Activity of Peroxynitrite May Aid Prevention and Control of Parkinson's Disease. International Journal of Molecular Sciences. 2020; 21: 3624. 
[12] Gazerani P. Probiotics for Parkinson's Disease. International Journal of Molecular Sciences. 2019; 20: 4121.

[13] Scuteri D, Rombolà L, Watanabe C, Sakurada S, Corasaniti MT, Bagetta G, et al. Impact of nutraceuticals on glaucoma: a systematic review. Progress in Brain Research. 2020; 257: 141-154.

[14] Bastounis A, Buckell J, Hartmann-Boyce J, Cook B, King S, Potter C, et al. The Impact of Environmental Sustainability Labels on Willingness-to-Pay for Foods: A Systematic Review and MetaAnalysis of Discrete Choice Experiments. Nutrients. 2021; 13: 2677.

[15] Prete R, Alam MK, Perpetuini G, Perla C, Pittia P, Corsetti A. Lactic Acid Bacteria Exopolysaccharides Producers: A Sustainable Tool for Functional Foods. Foods. 2021; 10: 1653.

[16] Drewnowski A. Impact of nutrition interventions and dietary nutrient density on productivity in the workplace. Nutrition Reviews. 2020; 78: 215-224.

[17] Meeusen R, Decroix L. Nutritional Supplements and the Brain. International Journal of Sport Nutrition and Exercise Metabolism. 2018; 28: 200-211.

[18] Pandareesh MD, Kandikattu HK, Razack S, Amruta N, Choudhari R, Vikram A, et al. Nutrition and Nutraceuticals in Neuroinflammatory and Brain Metabolic Stress: Implications for Neurodegenerative Disorders. CNS \& Neurological Disorders Drug Targets. 2018; 17: 680-688.

[19] Elumalai P, Lakshmi S. Role of Quercetin Benefits in Neurodegeneration. Advances in Neurobiology. 2016; 12: 229-245.

[20] López-Gómez L, Szymaszkiewicz A, Zielińska M, Abalo R. Nutraceuticals and Enteric Glial Cells. Molecules. 2021; 26: 3762.

[21] Rangarajan P, Karthikeyan A, Dheen ST. Role of dietary phenols in mitigating microglia-mediated neuroinflammation. NeuroMolecular Medicine. 2016; 18: 453-464.

[22] Verkhratsky A, Ho MS, Zorec R, Parpura V. The Concept of Neuroglia. Advances in Experimental Medicine and Biology. 2019; 1175: 1-13.

[23] von Bernhardi R, Eugenín-von Bernhardi J, Flores B, Eugenín León J. Glial Cells and Integrity of the Nervous System. Advances in Experimental Medicine and Biology. 2016; 949: 1-24.

[24] da Silva AB, Cerqueira Coelho PL, das Neves Oliveira M, Oliveira JL, Oliveira Amparo JA, da Silva KC, et al. The flavonoid rutin and its aglycone quercetin modulate the microglia inflammatory profile improving antiglioma activity. Brain, Behavior, and Immunity. 2020; 85: 170-185.

[25] Ma S, Fan L, Li J, Zhang B, Yan Z. Resveratrol promoted the M2 polarization of microglia and reduced neuroinflammation after cerebral ischemia by inhibiting miR-155. International Journal of Neuroscience. 2020; 130: 817-825.

[26] Ghasemi F, Bagheri H, Barreto GE, Read MI, Sahebkar A. Effects of Curcumin on Microglial Cells. Neurotoxicity Research. 2019; 36: $12-26$.

[27] Zhang J, Ren J, Liu Y, Huang D, Lu L. Resveratrol regulates the recovery of rat sciatic nerve crush injury by promoting the autophagy of Schwann cells. Life Sciences. 2020; 256: 117959.

[28] Yuan H, Zhang J, Liu H, Li Z. The protective effects of resveratrol on Schwann cells with toxicity induced by ethanol in vitro. Neurochemistry International. 2013; 63: 146-153.

[29] Zhao Z, Li X, Li Q. Curcumin accelerates the repair of sciatic nerve injury in rats through reducing Schwann cells apoptosis and promoting myelinization. Biomedicine \& Pharmacotherapy. 2017; 92: 1103-1110.

[30] Tello Velasquez J, Nazareth L, Quinn RJ, Ekberg JA, St John JA. Stimulating the proliferation, migration and lamellipodia of Schwann cells using low-dose curcumin. Neuroscience. 2016; 324: 140-150.

[31] Liu D, Liang XC, Sun Y, Wu YN, Zhang H. Combination of Quercetin, Hirudin and Cinnamaldehyde Promotes Schwann Cell Differentiation and Myelination against High Glucose by Inhibiting ERK Signaling Pathway. Chinese Journal of Integrative Medicine. 2020; 26: 591-598.

[32] Qu L, Liang X, Gu B, Liu W. Quercetin alleviates high glucoseinduced Schwann cell damage by autophagy. Neural Regeneration Research. 2014; 9: 1195-1203.

[33] de Souza SR, de Miranda Neto MH, Martins Perles JV, Vieira Frez FC, Zignani I, Ramalho FV, et al. Antioxidant Effects of the
Quercetin in the Jejunal Myenteric Innervation of Diabetic Rats. Frontiers in Medicine. 2017; 4: 8

[34] Ferreira P, Beraldi EJ, Borges SC, Natali M, Buttow NC. Resveratrol promotes neuroprotection and attenuates oxidative and nitrosative stress in the small intestine in diabetic rats. Biomedicine \& Pharmacotherapy. 2018; 105: 724-733.

[35] Seguella L, Gulbransen BD. Enteric glial biology, intercellular signalling and roles in gastrointestinal disease. Nature Reviews Gastroenterology \& Hepatology. 2021; 18: 571-587.

[36] Hanani M, Spray DC. Emerging importance of satellite glia in nervous system function and dysfunction. Nature Reviews Neuroscience. 2020; 21: 485-498.

[37] Hanani M. Role of satellite glial cells in gastrointestinal pain. Frontiers in Cellular Neuroscience. 2015; 9: 412.

[38] Hanani M. Satellite glial cells in sensory ganglia: from form to function. Brain Research Reviews. 2005; 48: 457-476.

[39] Christie K, Koshy D, Cheng C, Guo GF, Martinez JA, Duraikannu A, et al. Intraganglionic interactions between satellite cells and adult sensory neurons. Molecular and Cellular Neuroscience. 2015; 67: 1-12.

[40] Vit JP, Ohara PT, Bhargava A, Kelley K, Jasmin L. Silencing the Kir4.1 Potassium Channel Subunit in Satellite Glial Cells of the Rat Trigeminal Ganglion Results in Pain-Like Behavior in the Absence of Nerve Injury. Journal of Neuroscience. 2008; 28: 4161-4171.

[41] George D, Ahrens P, Lambert S. Satellite glial cells represent a population of developmentally arrested Schwann cells. GLIA. 2018; 66: 1496-1506.

[42] Miller KE, Richards BA, Kriebel RM. Glutamine-, glutamine synthetase-, glutamate dehydrogenase- and pyruvate carboxylaseimmunoreactivities in the rat dorsal root ganglion and peripheral nerve. Brain Research. 2002; 945: 202-211.

[43] Vit JP, Jasmin L, Bhargava A, Ohara PT. Satellite glial cells in the trigeminal ganglion as a determinant of orofacial neuropathic pain Neuron Glia Biology. 2006; 2: 247-257.

[44] Donegan M, Kernisant M, Cua C, Jasmin L, Ohara PT. Satellite glial cell proliferation in the trigeminal ganglia after chronic constriction injury of the infraorbital nerve. GLIA. 2013; 61: 20002008.

[45] Jager SE, Pallesen LT, Richner M, Harley P, Hore Z, McMahon S, et al. Changes in the transcriptional fingerprint of satellite glial cells following peripheral nerve injury. GLIA. 2020; 68: 1375-1395.

[46] Dublin P, Hanani M. Satellite glial cells in sensory ganglia: their possible contribution to inflammatory pain. Brain, Behavior, and Immunity. 2007; 21: 592-598.

[47] Hanani M, Huang TY, Cherkas PS, Ledda M, Pannese E. Glial cell plasticity in sensory ganglia induced by nerve damage. Neuroscience. 2002; 114: 279-283.

[48] Liu FY, Sun YN, Wang FT, Li Q, Su L, Zhao ZF, et al. Activation of satellite glial cells in lumbar dorsal root ganglia contributes to neuropathic pain after spinal nerve ligation. Brain Research. 2012; 1427: 65-77.

[49] Zhang H, Mei X, Zhang P, Ma C, White FA, Donnelly DF, et al. Altered functional properties of satellite glial cells in compressed spinal ganglia. GLIA. 2009; 57: 1588-1599.

[50] Jasmin L, Vit JP, Bhargava A, Ohara PT. Can satellite glial cells be therapeutic targets for pain control? Neuron Glia Biology. 2010; 6: 63-71.

[51] Takeda M, Takahashi M, Matsumoto S. Contribution of the activation of satellite glia in sensory ganglia to pathological pain. Neuroscience \& Biobehavioral Reviews. 2009; 33: 784-792.

[52] Jin SX, Zhuang ZY, Woolf CJ, Ji RR. P38 Mitogen-Activated Protein Kinase is Activated after a Spinal Nerve Ligation in Spinal Cord Microglia and Dorsal Root Ganglion Neurons and Contributes to the Generation of Neuropathic Pain. The Journal of Neuroscience. 2003; 23: 4017-4022.

[53] Obata K, Yamanaka H, Kobayashi K, Dai Y, Mizushima T, Katsura $\mathrm{H}$, et al. Role of Mitogen-Activated Protein Kinase Activation in Injured and Intact Primary Afferent Neurons for Mechanical and Heat Hypersensitivity after Spinal Nerve Ligation. Journal of Neuroscience. 2004; 24: 10211-10222.

[54] Freeman SE, Patil VV, Durham PL. Nitric oxide-proton stimulation of trigeminal ganglion neurons increases mitogen-activated 
protein kinase and phosphatase expression in neurons and satellite glial cells. Neuroscience. 2008; 157: 542-555.

[55] Zhuang ZY, Gerner P, Woolf CJ, Ji RR. ERK is sequentially activated in neurons, microglia, and astrocytes by spinal nerve ligation and contributes to mechanical allodynia in this neuropathic pain model. Pain. 2005; 114: 149-159.

[56] Li L, Zhou XF. Pericellular Griffonia simplicifolia I isolectin B4binding ring structures in the dorsal root ganglia following peripheral nerve injury in rats. The Journal of Comparative Neurology. 2001; 439: 259-274.

[57] Dominguez E, Rivat C, Pommier B, Mauborgne A, Pohl M. JAK/STAT3 pathway is activated in spinal cord microglia after peripheral nerve injury and contributes to neuropathic pain development in rat. Journal of Neurochemistry. 2008; 107: 50-60.

[58] Svensson CI, Schäfers M, Jones TL, Powell H, Sorkin LS. Spinal blockade of TNF blocks spinal nerve ligation-induced increases in spinal P-p38. Neuroscience Letters. 2005; 379: 209-213.

[59] Ji RR, Berta T, Nedergaard M. Glia and pain: is chronic pain a gliopathy? Pain. 2013; 154: S10-S28.

[60] Cecchini T, Ferri P, Ciaroni S, Cuppini R, Ambrogini P, Papa S, et al. Postnatal proliferation of DRG non-neuronal cells in vitamin E-deficient rats. The Anatomical Record. 1999; 256: 109-115.

[61] Nelson JS, Fitch CD, Fischer VW, Broun GO Jr, Chou AC. Progressive Neuropathologic Lesions in Vitamin E-Deficient Rhesus Monkeys. Journal of Neuropathology and Experimental Neurology. 1981; 40: 166-186.

[62] Liu Y, Ni Y, Zhang W, Sun YE, Ma Z, Gu X. N-acetyl-cysteine attenuates remifentanil-induced postoperative hyperalgesia via inhibiting matrix metalloproteinase-9 in dorsal root ganglia. Oncotarget. 2017; 8: 16988-17001.

[63] Hart AM, Terenghi G, Kellerth JO, Wiberg M. Sensory neuroprotection, mitochondrial preservation, and therapeutic potential of $\mathrm{N}$-acetyl-cysteine after nerve injury. Neuroscience. 2004; 125 : 91-101.

[64] Noorafshan A, Omidi A, Karbalay-Doust S, Aliabadi E, Dehghani F. Effects of curcumin on the dorsal root ganglion structure and functional recovery after sciatic nerve crush in rat. Micron. 2011; 42: 449-455.

[65] Jia T, Rao J, Zou L, Zhao S, Yi Z, Wu B, et al. NanoparticleEncapsulated Curcumin Inhibits Diabetic Neuropathic Pain Involving the P2Y12 Receptor in the Dorsal Root Ganglia. Frontiers in Neuroscience. 2018; 11: 755.

[66] Muto N, Matsuoka Y, Arakawa K, Kurita M, Omiya H, Taniguchi A, et al. Quercetin attenuates neuropathic pain in rats with spared nerve injury. Acta Medica Okayama. 2018; 72: 457-465.

[67] Yang R, Li L, Yuan H, Liu H, Gong Y, Zou L, et al. Quercetin relieved diabetic neuropathic pain by inhibiting upregulated P2X 4 receptor in dorsal root ganglia. Journal of Cellular Physiology. 2019; 234: 2756-2764.

[68] Yuan H, Ouyang S, Yang R, Li S, Gong Y, Zou L, et al. Osthole alleviated diabetic neuropathic pain mediated by the $\mathrm{P} 2 \mathrm{X} 4$ receptor in dorsal root ganglia. Brain Research Bulletin. 2018; 142: 289296.

[69] Wu B, Ma Y, Yi Z, Liu S, Rao S, Zou L, et al. Resveratrol-decreased hyperalgesia mediated by the $\mathrm{P} 2 \mathrm{X} 7$ receptor in gp120-treated rats. Molecular Pain. 2017; 13: 174480691770766.

[70] Xie J, Liu S, Wu B, Li G, Rao S, Zou L, et al. The protective effect of resveratrol in the transmission of neuropathic pain mediated by the P2X7 receptor in the dorsal root ganglia. Neurochemistry International. 2017; 103: 24-35.

[71] Demirkol O, Adams C, Ercal N. Biologically Important Thiols in Various Vegetables and Fruits. Journal of Agricultural and Food Chemistry. 2004; 52: 8151-8154.

[72] Aldini G, Altomare A, Baron G, Vistoli G, Carini M, Borsani L, et al. N-Acetylcysteine as an antioxidant and disulphide breaking agent: the reasons why. Free Radical Research. 2018; 52: 751-762.

[73] Schwalfenberg GK. N-Acetylcysteine: a Review of Clinical Use- fulness (an Old Drug with New Tricks). Journal of Nutrition and Metabolism. 2021; 2021: 9949453.

[74] Van Doren SR. Matrix metalloproteinase interactions with collagen and elastin. Matrix Biology. 2015; 44-46: 224-231.

[75] Gu HW, Xing F, Jiang MJ, Wang Y, Bai L, Zhang J, et al. Upregulation of matrix metalloproteinase- $9 / 2$ in the wounded tissue, dorsal root ganglia, and spinal cord is involved in the development of postoperative pain. Brain Research. 2019; 1718: 64-74.

[76] Liu YC, Berta T, Liu T, Tan PH, Ji RR. Acute Morphine Induces Matrix Metalloproteinase-9 up-Regulation in Primary Sensory Neurons to Mask Opioid-Induced Analgesia in Mice. Molecular Pain. 2012; 8: 19

[77] Crowley LC, Marfell BJ, Waterhouse NJ. Detection of DNA Fragmentation in Apoptotic Cells by TUNEL. Cold Spring Harbor Protocols. 2016; 2016.

[78] Menon VP, Sudheer AR. Antioxidant and anti-inflammatory properties of curcumin. Advances in Experimental Medicine and Biology. 2007; 595: 105-125.

[79] Abrahams S, Haylett WL, Johnson G, Carr JA, Bardien S. Antioxidant effects of curcumin in models of neurodegeneration, aging, oxidative and nitrosative stress: a review. Neuroscience. 2019; 406: $1-21$.

[80] Schmelzer JD, Zochodne DW, Low PA. Ischemic and reperfusion injury of rat peripheral nerve. Proceedings of the National Academy of Sciences of the United States of America. 1989; 86: 1639-1642.

[81] Wu MY, Yiang GT, Liao WT, Tsai AP, Cheng YL, Cheng PW, et al. Current Mechanistic Concepts in Ischemia and Reperfusion Injury. Cellular Physiology and Biochemistry. 2018; 46: 1650-1667.

[82] Ferrari RS, Andrade CF. Oxidative Stress and Lung IschemiaReperfusion Injury. Oxidative Medicine and Cellular Longevity. 2015; 2015: 590987.

[83] Oskouie MN, Aghili Moghaddam NS, Butler AE, Zamani P, Sahebkar A. Therapeutic use of curcumin-encapsulated and curcumin-primed exosomes. Journal of Cellular Physiology. 2019; 234: 8182-8191.

[84] Boots AW, Haenen GR, Bast A. Health effects of quercetin: from antioxidant to nutraceutical. European Journal of Pharmacology. 2008; 585: 325-337.

[85] Jafarinia M, Sadat Hosseini M, kasiri N, Fazel N, Fathi F, Ganjalikhani Hakemi M, et al. Quercetin with the potential effect on allergic diseases. Allergy, Asthma \& Clinical Immunology. 2020; 16: 36.

[86] Costa LG, Garrick JM, Roquè PJ, Pellacani C. Mechanisms of Neuroprotection by Quercetin: Counteracting Oxidative Stress and more. Oxidative Medicine and Cellular Longevity. 2016; 2016: 2986796.

[87] Haleagrahara N, Miranda-Hernandez S, Alim MA, Hayes L, Bird G, Ketheesan N. Therapeutic effect of quercetin in collageninduced arthritis. Biomedicine \& Pharmacotherapy. 2017; 90: 3846.

[88] Cesarone MR, Belcaro G, Hu S, Dugall M, Hosoi M, Ledda A, et al. Supplementary prevention and management of asthma with quercetin phytosome: a pilot registry. Minerva Medica. 2019; 110: 524-529.

[89] Galiniak S, Aebisher D, Bartusik-Aebisher D. Health benefits of resveratrol administration. Acta Biochimica Polonica. 2019; 66: $13-21$.

[90] Lee BH, Kushwah R, Wu J, Ng P, Palaniyar N, Grinstein S, et al. Adenoviral vectors stimulate innate immune responses in macrophages through cross-talk with epithelial cells. Immunology Letters. 2010; 134: 93-102.

[91] Erny D, Hrabě de Angelis AL, Prinz M. Communicating systems in the body: how microbiota and microglia cooperate. Immunology. 2017; 150: 7-15.

[92] Erny D, Prinz M. How microbiota shape microglial phenotypes and epigenetics. GLIA. 2020; 68: 1655-1672. 